\title{
Fluorescence Images of DNA-Bound YOYO between Coupled Silver Particles
}

\author{
Jian Zhang, Yi Fu, and Joseph R. Lakowicz \\ Center for Fluorescence Spectroscopy, University of Maryland School of Medicine, Department of \\ Biochemistry and Molecular Biology, 725 West Lombard Street, Baltimore, Maryland 21201
}

\section{Abstract}

Oligonucleotide-bound silver particles were coupled through hybridization with target complementary oligonucleotides. YOYO molecules were intercalated into DNA duplexes bound between the coupled metal particles. Fluorescence images of YOYO molecules were monitored by scanning confocal microscopy. Relative to the free single YOYO, the emission brightness of the image was enhanced 80-fold after intercalating the fluorophores into the DNA duplexes between the coupled silver particles. Some images of the labeled metal particle dimers were observed to be dumbbell-shaped, suggesting that the stretching of intercalated YOYO molecules was restricted because of the orientation effect of fluorophores. The shortened lifetime of YOYO molecules between the coupled metal particles indicated that the fluorescence was enhanced via a near-field interaction mechanism between the fluorophore and the metal nanoparticle.

\section{Introduction}

A fluorophore near a metal surface usually behaves as an oscillating dipole to radiate energy when fluorescence occurs. ${ }^{1,2}$ The fluorescence thus can be enhanced by a local electric field that exists near a metal particle induced by incident light through a near-field interaction between the fluorophore and metal particle, which is defined as metal-enhanced fluorescence (MEF). ${ }^{3}$ The local electric field near the metal particle can be calculated by the finite-difference time-domain (FDTD) technique. ${ }^{4}$ When the metal particles are in close proximity to each other, the local electric field between the metal particles becomes more intensive, so the fluorophores localized in the overlapped field are expected to be influenced more strongly and result in a larger scale of fluorescence enhancement. ${ }^{5}$ This effect has been demonstrated by our recent experimental and theoretical results. ${ }^{6}$

The brightness or emission rate of the fluorophore determines its observability, and the photostability determines the observation time. The use of fluorophores coupling with metal nanoparticles can significantly improve the detection sensitivity in the clinical and biological detections. ${ }^{7,8}$ Considering that the fluorescence can be enhanced when the fluorophores near the surfaces of metal substrates are localized, we suggest that the detection sensitivity can be further improved when using MEF to assist imaging during the measurement. ${ }^{9}$ Experimentally, the silver particles were synthesized with suitable sizes, ${ }^{10}$ and the single-stranded

(C) 2007 American Chemical Society

*Corresponding author. lakowicz@cfs.umbi.umd.edu..

Supporting Information Available: Fluorescence images of YOYO intercalated in free DNA duplexes in the absence of metal and DNA duplexes bound between coupled silica beads and coupled silver particles, fluorescence images and corresponding histograms of unbound ethidium and ethidium intercalated into unbound DNA duplexes in the absence of metal and between silver particles, and chemical structures of YOYO and ethidium. This material is available free of charge via the Internet at http://pubs.acs.org. 
oligonucleotide chains were covalently bound to the metal particles via surface reactions in this case. ${ }^{11}$ These oligonucleotide-bound metal particles were coupled as metal dimers by hybridization with target oligonucleotides in solution ${ }^{4 \mathrm{a}}$ and further labeled through the intercalation of YOYO molecules into the bound duplexes that exist between the coupled metal particles. ${ }^{12}$ The fluorescence images were recorded by scanning confocal microscopy and investigated as compared with the free YOYO molecules or the intercalated YOYO molecules in the absence of metal.

\section{Experimental Section}

All reagents and spectroscopic grade solvents were used as received from Fisher or Aldrich. A regenerated cellulose (RC) dialysis membrane (MWCO 50000 ) was obtained from Spectrum Laboratories, Inc. Nanopure water $(>18.0 \mathrm{M} \Omega \cdot \mathrm{cm})$ purified using a Millipore Milli-Q gradient system was used in all experiments. (2-Mercapto-propionylamino) acetic acid 2,5-dioxopyrrolidin-1-ylester was synthesized as previous reported. Oligonucleotides were synthesized by the Biopolymer Laboratory at the University of Maryland at Baltimore.

\section{Preparing Tiopronin-Coated Metal Nanoparticles and Binding Aminated Single-Stranded Oligonucleotides}

Tiopronin-coated silver nanoparticles were prepared using a modified Brust reaction with a tiopronin/silver nitrate mole ratio of 1/6 in methanol using reduction by an excess amount of sodium borohydride. ${ }^{13}$ After filtration, the residual precipitated particles were washed thoroughly with methanol and acetone. The particles $(1 \mathrm{mg} / \mathrm{mL})$ and tiopronin $(10 \mathrm{mM})$ were then co-dissolved in water, and the solution was stirred for $24 \mathrm{~h}$ for annealing of the particles. 14 The water was removed under vacuum, and the residual was washed thoroughly with methanol and acetone. The residual solid tiopronin-coated particles were further purified by dialysis against water.

The tiopronin-coated silver particles were succinimidylated by a ligand exchange reaction. ${ }^{15}$ (2-Mercapto-propionylamino) acetic acid 2,5-dioxo-pyrrolidin-1-ylester $\left(1 \times 10^{-8} \mathrm{M}\right)$ and tiopronin-coated silver particles $\left(1 \mathrm{mg} / \mathrm{mL}, 1 \times 10^{-8} \mathrm{M}\right)$ were co-dissolved in a mixing solvent of water/methanol $(\mathrm{v} / \mathrm{v}=1 / 1)$ and stirred for $24 \mathrm{~h}$. The suspension was removed by centrifugation at $8000 \mathrm{rpm}$. The residue was washed by methanol and water, respectively, and then dispersed in water at $\mathrm{pH}=7$.

\section{Preparing Silica Beads and Binding Aminated Single-Stranded Oligonucleotides}

Monodispersed silica beads were prepared using the Stöber method with $1.4 \times 10^{-2} \mathrm{M}$ tetraethyl orthosilicate in $50 \mathrm{~mL}$ of ethanol. ${ }^{16} 1.0 \mathrm{~mL}$ of $30 \%$ ammonia alcohol solution was added dropwise under vigorous stirring. The solution became turbid after stirring overnight because of the formation of silica beads. The mixture was centrifuged to remove the suspension, and the residual was washed thoroughly with ethanol. The beads were redispersed in $50 \mathrm{~mL}$ of ethanol and aminated by adding $10 \mu \mathrm{L}$ of 3-amino-propyltrimethoxy silane with continuous stirring for $5 \mathrm{~h} .{ }^{17}$ The aminated beads were centrifuged, washed with alcohol and water, and then dispersed in $10 \mathrm{~mL}$ of water. The ligands on the metal particles were succinimidylated by co-dissolving an excess amount of hexanic acid bis( $N$-succinimidyl) ester at a molar ratio of $1 / 20$. The solution was stirred for $4 \mathrm{~h}$. The suspension was removed by centrifugation at 8000 $\mathrm{rpm}$. The residue was washed by water and then dispersed in water at $\mathrm{pH}=7.0$.

\section{Binding DNA on the Particles and Coupling Them by Hybridization with Target DNA}

The aminated oligonucleotides (Scheme 1) were chemically bound onto the silica or silver particles by adding oligonucleotides $(1 \mu \mathrm{M})$ to the succinimidylated particles $(1 \mathrm{mg} / \mathrm{mL})$ in water with continuous stirring for $24 \mathrm{~h} .{ }^{18}$ The suspension was removed by centrifugation at 
$8000 \mathrm{rpm}$. The residue was washed by water and then dispersed in $50 \mathrm{mM}$ PBS buffer solution at $\mathrm{pH}=7.2$. The DNA-bound particles were further purified by extensive dialysis against buffer solution to remove all free impurities (MWCO 50000 ). The hybridizations were performed at a particle/target oligonucleotide molar ratio of $1 / 1$ for $24 \mathrm{~h}$. The suspension was removed by centrifugation at $8000 \mathrm{rpm}$. The residual particles were washed with buffer solution.

\section{Labeling the Coupled Metal Particles by YOYO Intercalation}

A $10 \mu \mathrm{L}$ portion of $0.1 \mathrm{mM}$ YOYO solution was added to $1.0 \mathrm{~mL}$ of buffer solution containing the coupled silica or metal particles, and then incubated overnight. ${ }^{19}$ Because the fluorophore was largely an excess amount, the DNA duplexes between the coupled particles were supposed to be saturate-labeled by the YOYO molecules. The suspension was removed by centrifugation at $8000 \mathrm{rpm}$. The residual particles were washed with buffer solution. These dye-labeled samples were further purified by dialysis against buffer solution to remove all free fluorophores (MWCO 50 000), and then used directly for fluorescence image measurements. Ethidium was also used instead of YOYO to label the DNA duplexes by the same method.

\section{Spectra, Lifetime, and TEM Measurements}

Absorption spectra were monitored with a Hewlett-Packard 8453 spectrophotometer. Ensemble fluorescence spectra were recorded in solution with a Cary Eclipse Fluorescence Spectrophotometer. All fluorescence image studies were performed using a time-resolved confocal microscope (MicroTime 200, PicoQuant). Immobilization of nanoparticles on glass coverslips was achieved by adding $20 \mu \mathrm{L}$ of 100 -fold diluted nanoparticle suspension onto an amino-silanized coverslip following by spin drying at $4000 \mathrm{rpm}$. A single-mode pulsed laser diode (470 nm, $100 \mathrm{ps}, 40 \mathrm{MHz}$ ) (PDL800, PicoQuant) was used as the excitation light. The collimated laser beam was spectrally filtered by an excitation filter (D467/10, Chroma) before being directing into an inverted microscope (Olympus, IX 71). An oil immersion objective (Olympus, 100 $\times 1.3 \mathrm{NA}$ ) was used for both focusing laser light onto the sample and collecting fluorescence emission from the sample. The fluorescence that passed a dichroic was focused onto a $75 \mu \mathrm{m}$ pinhole for spatial filtering to reject out-of-focus signals, and then reached the single photon avalanche diode (SPAD) (SPCM-AQR-14, Perkin-Elmer, Inc.). Images were recorded by raster scanning (in a bidirectional fashion) the sample over the focused spot of the incident laser with a pixel integration of $0.6 \mathrm{~ms}$. The excitation power into the microscope was maintained at less than $1 \mu \mathrm{W}$. Time-dependent fluorescence data were collected with a dwell time of $50 \mathrm{~ms}$. The data were stored in time-tagged-time-resolved (TTTR) mode, which allows recording every detected photon with its individual timing information. Instrument response function (IRF) widths of about $300 \mathrm{ps}$ full width at half-maximum (fwhm) could be obtained in combination with a pulsed diode laser, which permits the recording of sub-nanosecond fluorescence lifetimes extendable to less than $100 \mathrm{ps}$ with reconvolution. Lifetimes were estimated by fitting to a $\chi^{2}$ value of less than 1.2 and with a residuals trace that was fully symmetrical about the zero axis. All measurements were performed in a dark compartment at room temperature.

Transmission electron micrographs (TEMs) were taken with a side-entry Philips electron microscope at $120 \mathrm{keV}$. Samples were cast from water solutions onto standard carbon-coated (200-300 ̊̊) Formvar films on copper grids (200 mesh) by placing a droplet of a $1 \mathrm{mg} / \mathrm{mL}$ aqueous sample solution on the grids. The size distribution of the metal core was analyzed with Scion Image Beta Release 2, counting at least 200 particles.

\section{Results and Discussion}

In the experiments, the silver particles were prepared by the reduction of silver nitrate in water, protected by tiopronin monolayer, and succinimidylated via a ligand exchange reaction. ${ }^{18}$ The 
aminated oligonucleotides were then covalently bound to the succinimidylated silver particle via condensation (Scheme 1) ${ }^{17}$ It was shown that the citrate-coated silver particles displayed a metal plasmon absorbance at $401 \mathrm{~nm}$ in water (Figure 1). The average diameter of silver cores was estimated to be $20 \mathrm{~nm}$ from TEM images (Figure 2A). The surface reactions on the silver particles did not significantly alter the absorbance spectrum and the TEM images of silver particles because only a few ligands were involved.

The silver particles were coupled through the hybridization of bound oligonucleotides with the target oligonucleotides in $50 \mathrm{mM}$ phosphate buffered saline (PBS) buffer solution at $\mathrm{pH}=7.4$ by the molar ratio of $1 / 2$ (Scheme 1). ${ }^{4 a}, 18$ Because there was approximately one single bound oligonucleotide on each metal particle, the hybridization was mostly assumed to result in the dimerized particles. The coupled metal particles were fluorescently labeled by the intercalated YOYO in the DNA duplexes between the metal dimers. ${ }^{12}$ The coupled metal particles displayed a slight red-shifting of absorbance to $408 \mathrm{~nm}$ (Figure 1), consistent with the result of Mirkin et al. ${ }^{11 a}$ No absorbance from the intercalated fluorophore could be detected because of the overlap from the strong plasmon resonance of the silver particles. This coupling of metal particles was also confirmed by TEM images (Figure 2B), on which about $60 \%$ of the metal particles were observed to exist as monomers without coupling, and $40 \%$ were observed as dimers. There were few metal particles showing as aggregates. The DNA duplexes were fluorescently labeled by YOYO molecules via intercalations. ${ }^{12}$ Consequently, only the coupled metal particles could be fluorescently labeled because of the formation of DNA duplexes between them. The particle monomers could not be labeled in this case because of the absence of the DNA duplex. Hence, we did not further separate the monomers from the dimers.

Alternatively, we also tried to directly bind the YOYO intercalated DNA duplexes on the metal particles. But the result showed that the labeled DNA duplexes almost could not be bound to the metal particles, probably because of the steric hindrance of bulky double-stranded DNA and the strong repulsion by the deprotonated surfaces of the metal particles.

The solution that contained the labeled metal particles was spin-cast on the aminated glass coverslips for the fluorescence imaging measurements using scanning confocal microscopy. We first recorded the fluorescence images of labeled DNA duplexes in the absence of metal particles as a control. YOYO is known to display an emission maximum at $509 \mathrm{~nm}$ upon excitation at $491 \mathrm{~nm}$ in water. ${ }^{19}$ The free YOYO samples showed relatively dim and round spots (Figure 3A) with $470 \mathrm{~nm}$ laser line excitation. When intercalated into the DNA duplexes, the well-defined spots became larger and brighter (Figure 3B and additional images in Figure S1, Supporting Information). The larger size of the spots was probably due to the overlapping of emission from multiple-intercalated fluorophores in one DNA duplex. The round spots were about 4 times brighter when interacting YOYO into DNA duplexes. This value was much lower than that reported (1000-fold) in solution, which is most probably due to the different medium surrounding for the intercalated fluorophores in solution or in the solid state.

Most of isolated single fluorophores display a typical one-step photobleaching. ${ }^{9 a}$ In this study, the time trace for the labeled DNA duplex showed a gradual decay, indicating that there were multiple fluorophores existing in one DNA duplex (Figure 4). ${ }^{7}$ According to Larsson et al., ${ }^{19 a}$ each intercalated YOYO molecule occupies $4-5$ bps of DNA duplex chain, so one DNA duplex was estimated to contain about 10 intercalated YOYO molecules. This result is consistent with the time-dependent intensity studies.

Next, we investigated the emission behavior of fluorophores when intercalated into the DNA duplexes bound between the coupled metal particles. It was shown that the image spots with the metal particles (Figure 5) became even larger and brighter than those in the absence of metal (Figure 3B). More than 100 images were collected to compile brightness histograms (Figure 6). The apparent intensity displayed a 20 -fold increase compared with the YOYO- 
intercalated DNA in the absence of metal, and an 80-fold increase with the free single fluorophore. We did not count the accurate number of fluorophores in each DNA duplex between the metal particles; however, this number was assumed to be lower than that in the unbound DNA duplex in the absence of metal because of a steric hindrance from the metal particles. Thus, the fluorescence enhancement should not be ascribed to the number of fluorophores intercalated into the DNA duplexes. In order to exclude the effect of metallic scattering on the brightness enhancement, we examined the scattering background of the coupled metal particles without the YOYO intercalation, showing that the scattering signals were particularly weak and did not contribute significantly to the enhanced brightness (Figure 3C). Thus, we confirm that the fluorescence of intercalated YOYO between the coupled particles was indeed enhanced as a result of the MEF mechanism. ${ }^{3 a}$ This mechanism was furthermore verified by a coupled model using the silica beads instead of the metal particles. We synthesized the silica beads that were similar in size to the metal particles used in the experiments. These silica beads were bound chemically with the same single-stranded oligonucleotides, coupled by hybridization with the target DNA, and labeled by the YOYO intercalation as described in the experimental section. It was shown that, relative to the free single YOYO molecules, the image spots from the labeled silica beads became larger like the labeled metal particles, but the intensity level was close to that of the labeled DNA duplex in the absence of metal (Figure 3d and additional images in Figure S2, Supporting Information), indicating that the metal substrate played a key role in the fluorescence enhancement. We also examined the mixture of free YOYO and freshly prepared silver particles without DNA duplexes in solution. The intensities of the emission spectra were observed to be close to those from free single YOYO molecules, indicating that the fluorescence was enhanced significantly only when the fluorophores were localized near the metal particles.

We noticed that the shapes of fluorescence spots from the intercalated YOYO molecules appear varied depending on whether the DNA duplexes were bound on silica beads or silver particles. The free YOYO molecules and labeled DNA duplexes in the absence of particles display roughly round, well-defined shapes, as commonly observed for fluorophores (Figure 1a,b), while, when the labeled DNA duplexes were bound on the coupled silica beads (Figure 3D and additional images in Figure S2, Supporting Information) or the coupled silver particles (Figure 5 ), the observed spots were extended and split in a dumbbell shape. We examined more than 100 different molecules from the recorded images. Nearly half of the observed spots were configured as dumbbell-shaped. We also investigated those round spots using a defocusing method. A comprehensive series of defocused images were obtained by defocusing the image some distance from the objective plane using the microscope adjustment along the optic axis. The focus adjustment was calibrated by comparing image intensities of $100 \mathrm{~nm}$ dye-doped latex spheres. The highest fluorescence intensity was achieved with the beam waist in the center of the sphere, that is, around $100 \mathrm{~nm}$ above the glass/air interface. The distances away from the focus planes are given in the figure captions. The images (Figure 7 and additional images in Figure S3, Supporting Information) showed that some round spots could take on a dumbbell shape after defocusing. The percentage of dumbbell-shaped configuration decreased to $20 \%$ when the labeled DNA molecules were bound between the coupled silica beads. This value was lower than that between the coupled metal particles. We do not know the exact reason, but it appears that the dumbbell-shaped configurations were from the oriented fluorophore. 20,21 We assume that the binding of particles at the ends of DNA duplexes can stretch the DNA molecules and further orient the intercalated fluorophores in the DNA duplexes. It is known that a fluorophore can be regarded as a radiative dipole and gives an anisotropic emission with a polarized excitation. Because the dumbbell-shaped images occur only when the fluorophore orients parallel to the surface of observation, ${ }^{20}$ the intercalated fluorophores exist as the most stable states that are parallel to the sample surface. We also found that the change in the polarization of the incident source did not significantly alter the shape of the images (Figure S4, Supporting Information), further verifying the perpendicular orientation of fluorophores. 
Meanwhile, some fluorophores also exist parallel to the sample surface, which corresponds to our defocused images shown in Figure 7 and Figure S3 (Supporting Information).

Instead of YOYO, we also employed ethidium, which can intercalate into DNA duplexes in a similar mechanism, to investigate the orientation effect of fluorophores between the coupled metal particles. The intercalations of fluorophores and measurements of fluorescence images for the ethidium were carried out in the same method as for the YOYO molecules. The results revealed that the brightness of the image was enhanced 40-fold relative to the free single ethidium molecules (Figure S5, Supporting Information). This efficiency is lower than that of YOYO. In addition, the images of the ethidium molecules displayed only the regular round images without splitting into the dumbbell shape like YOYO. Herein, we have to analyze the different orientation effects of YOYO and ethidium molecules from their chemical structures (Figure S6, Supporting Information). The YOYO molecule is a long-rod bisintercalator, but the ethidium molecule is a small, round monointercalator. Hence, we believe that the YOYO molecules were intercalated more strongly and densely into the DNA duplexes than the ethidium molecules, and the stretching of these intercalated YOYO molecules was restricted more strictly when the fluorophores were intercalated in the DNA duplexes between the coupled metal dimers. The difference in their intercalations with the DNA duplexes was verified by molecular elasticity measurements. ${ }^{22}$ A slower stretching of the fluorophores can lead to a strong orientation effect for the fluorescence image measurement. Hence, the strongly intercalated YOYO molecules between the coupled metal particles are expected to display a well-oriented emission, but the weakly intercalated ethidium molecules do not display a significant orientation effect.

Fluorescence near metal particles is enhanced via an increase in the intrinsic decay rate. ${ }^{1,3}$ As a result, the lifetime for a bound fluorophore near the metal surface should be shortened. In this case, the lifetime data were derived from the time traces using time-correlated single photon counting (TCSPC) and analyzed in terms of single-exponential decays (Figure 8). It was shown that the lifetime is slightly shortened from $4.3 \mathrm{~ns}$ (free YOYO molecules) to $3.6 \mathrm{~ns}$ (intercalated YOYO molecules in the absence of metal), which is consistent with the change in brightness. The lifetime is not altered much when binding the labeled DNA duplexes to the silica beads (3.3 ns) but is shortened dramatically when binding the DNA duplexes to the coupled metal particles ( $1.6 \mathrm{~ns})$, verifying the strong interactions between the fluorophores and metal particles. An 80-fold enhancement of a confocal image shows that the target DNA can be detected at the single DNA molecule level.

Simultaneously, the photostability was influenced by the lifetime of fluorophores near metal particles because of the greater number of excitation-emission cycles prior to photobleaching. $3 \mathrm{~b}$ The time traces indicated that about $1 / 3$ of the labeled metal dimers were still emitting photons after $30 \mathrm{~s}$, but most of the free YOYO molecules were photobleached after $2 \mathrm{~s}$, and the intercalated YOYO molecules in the absence of metal particles or in the presence of silica beads can last for about $5 \mathrm{~s}$, as depicted in Figure 4. These results indicate that the photostability of fluorophore was extended 10 -fold on the coupled metal particles.

\section{Summary}

In this paper, we studied the fluorescence images for fluorophores intercalated in DNA duplexes between coupled metal particles. The oligonucleotide-bound silver particles were coupled by hybridization with the target DNA, and YOYO molecules were interacted into duplex DNA chains between the coupled metal particles. Fluorescence images were recorded using scanning confocal microscopy. It was shown that the fluorescence brightness from the fluorophores intercalated into the silver particle-bound DNA duplexes was enhanced 80-fold as compared with that of the free single YOYO molecules because of the near-field interaction 
of fluorophores with the plasmon resonance from the sub-wavelength size metal particles. It was interesting to notice that the images of YOYO molecules intercalated into the silver particle-bound DNA duplexes displayed a dumbbell shape, implying that the stretching of DNA duplexes was restricted between the metal particles and that the intercalated fluorophores in the DNA duplexes were well-orientated. The lifetime of YOYO in the DNA duplex between the coupled metal particles was shortened, suggesting that the fluorescence was enhanced via the near-field interaction mechanism of fluorophore with the metal substrate.

\section{Supplementary Material}

Refer to Web version on PubMed Central for supplementary material.

\section{Acknowledgment}

This research was supported by a grant from NIH (HG-00255) and a grant from NCRR (RR-08119).

\section{References}

1. Lakowicz, JR. Principles of Fluorescence Spectroscopy. Vol. 3rd ed.. Kluwer Academic/Plenum Publishing; New York: 2006.

2. (a) Yonzon CR, Jeoung E, Zou S, Schatz GC, Mrksich M, Van, Duyne RP. J. Am. Chem. Soc 2004;126:12669. [PubMed: 15453801] (b) Yu F, Persson B, Lofas S, Knoll W. J. Am. Chem. Soc 2004;126:8902. [PubMed: 15264814] (c) Kumbhar AS, Kinnan MK, Chumanov G. J. Am. Chem. Soc 2005;127:12444. [PubMed: 16144364]

3. Lakowicz JR. Anal. Biochem 2001;298:1. [PubMed: 11673890] (b) Lakowicz JR. Anal. Biochem 2005;337:171. [PubMed: 15691498]

4. Taflove, A.; Hagness, SC. Computational Electrodynamics: The Finite-Difference Time-Domain Method. Artech House; Boston: 2000. Sullivan, DM. Electromagnetic Simulation Using the FDTD Method. IEEE Press; New York: 2000. (c) Gray SK, Kupka T. Phy. Rev. B 2003;68:045415. (d) Chang S-H, Gray SK, Schatz GC. Opt. Express 2005;13:3150. [PubMed: 19495214]

5. (a) Kelly KL, Coronado E, Zhao LL, Schatz GC. J. Phys. Chem. B 2003;107:668. (b) Hao E, Li S, Bailey RC, Zou S, Schatz GC, Hupp JT. J. Phys. Chem. B 2004;108:1224.

6. Zhang J, Fu Y, Chowdhury MH, Lakowicz JR. Nano Lett 2007;7:2101. [PubMed: 17580926]

7. Nie, S. Single-Molecule Optical Detection, Imaging and Spectroscopy. Basche, T.; Moerner, WE.; Orrit, M.; Wild, UP., editors. Wiley/VCH; New York: 1997. (b) Ambrose WP, Goodwin PM, Jett JH, Van Orden A, Werner JH, Keller RA. Chem. Rev 1999;99:2929. [PubMed: 11749506] (c) Bokinsky G, Zhuang X. Acc. Chem. Res 2005;38:566. [PubMed: 16028891]

8. (a) Li Q, Seeger S. Anal. Chem 2006;78:2732. [PubMed: 16615786] (b) Allen MW, Urbauer RJB, Johnson CK. Anal. Chem 2004;76:3630. [PubMed: 15228334] (c) Trabesinger W, Schutz GJ, Gruber HJ, Schindler H, Schmidt T. Anal. Chem 1999;71:279. [PubMed: 9921136]

9. (a) Fu Y, Lakowicz JR. Anal. Chem 2006;78:6238. [PubMed: 16944907] (b) Fu Y, Lakowicz JR. J. Phys. Chem. B 2006;110:22557. [PubMed: 17092001]

10. Feldheim, DL.; Foss, CA. Metal Nanoparticles: Synthesis, Characterization and Applications. Marcel Dekker, Inc.; New York: 2002. Kreibig, U.; Vollmer, M. Optical Properties of Metal Clusters. Springer-Verlag; Berlin and Heidelberg, Germany: 1995. Hayat, MA., editor. Colloidal Gold: Principles, Methods, and Applications. Academic Press; San Diego, CA: 1991.

11. (a) Rosi NL, Mirkin CA. Chem. Rev 2005;105:1547. [PubMed: 15826019] (b) Zhang J, Malicka J, Gryczynski I, Lakowicz JR. J. Phys. Chem. B 2005;109:7643. [PubMed: 16851886]

12. (a) Wang G, Zhang J, Murray RW. Anal. Chem 2002;74:4320. [PubMed: 12236338] (b) Tse WC, Boger DL. Acc. Chem. Res 2004;38:61. [PubMed: 14730995]

13. (a) Brust M, Walker M, Bethell D, Schiffrin DJ, Whyman R. J. Chem. Soc., Chem. Commun 1994:801. (b) Huang T, Murray RW. Langmuir 2002;18:7077. (c) Huang T, Murray RW. J. Phys. Chem. B 2001;105:12498. 
14. (a) Hicks JF, Miles DT, Murray RW. J. Am. Chem. Soc 2002;124:13322. [PubMed: 12405861] (b) Schaaff TG, Shafigullin MN, Khoury JT, Vezmar I, Whetten RI. J. Phys. Chem. B 2001;105:8785.

15. (a) Templeton AC, Wuelfing WP, Murray RW. Acc. Chem. Res 2000;33:27. [PubMed: 10639073] (b) Ingram RS, Hostetler MJ, Murray RW. J. Am. Chem. Soc 1997;119:9175.

16. Stober W, Fink A, Bohn E. J. Colloid Interface Sci 1968;26:62.

17. (a) Zhang J, Fu Y, Lakowicz JR. J. Chem. Phys. C 2007;111:1955. (b) Zhang J, Gryczynski I, Gryczynski Z, Lakowicz JR. J. Chem. Phys. B 2006;110:8986.

18. (a) Zhang J, Fu Y, Chowdhury MH, Lakowicz JR. J. Chem. Phys. C 2007;111:11784. (b) Zhang J, Fu Y, Lakowicz JR. J. Chem. Phys. C 2007;111:50.

19. (a) Larsson A, Carlsson C, Jonsson M, Albinsson B. J. Am. Chem. Soc 1994;116:8459. (b) Shimizu M, Sasaki S, Tsuruoka M. Biomacromolecules 2005;6:2703. [PubMed: 16153109]

20. (a) Dickson RM, Norris DJ, Moener WE. Phys. Rev. Lett 1998;24:5322. (b) Bartko AP, Dickson RM. J. Phys. Chem. B 1999;103:11237.Novotny, L.; Hecht, B. Principle of Nano-optics. Cambridge University Press; Cambridge, U.K.: 2006.

21. (a) Failla AV, Qian H, Qian H, Hartschuh A, Meixner AJ. Nano Lett 2006;6:1374. [PubMed: 16834414] (b) Schroeyers W, Vallee R, Patra D, Hofkens J, Habuchi S, Vosch T, Cotlet M, Mullen K, Enderlein J, De Schryver FC. J. Am. Chem. Soc 2004;126:14310. [PubMed: 15521723] (c)

Bohmer M, Enderlein J. J. Opt. Soc. Am. B 2003;20:554. (d) Patra D, Gregor I, Enderlein J. J. Phys. Chem. A 2004;108:6836. (e) Toprak E, Enderlein J, Syed S, McKinney SA, Petschek RG, Ha T, Goldman YE, Selvin PR. Proc. Natl. Acad. Sci. U.S.A 2006;103:6495. [PubMed: 16614073]

22. Sischka A, Toensing K, Eckel R, Wilking S,D, Sewald N, Ros R, Anselmetti D. Biophy. J 2005;88:404. 


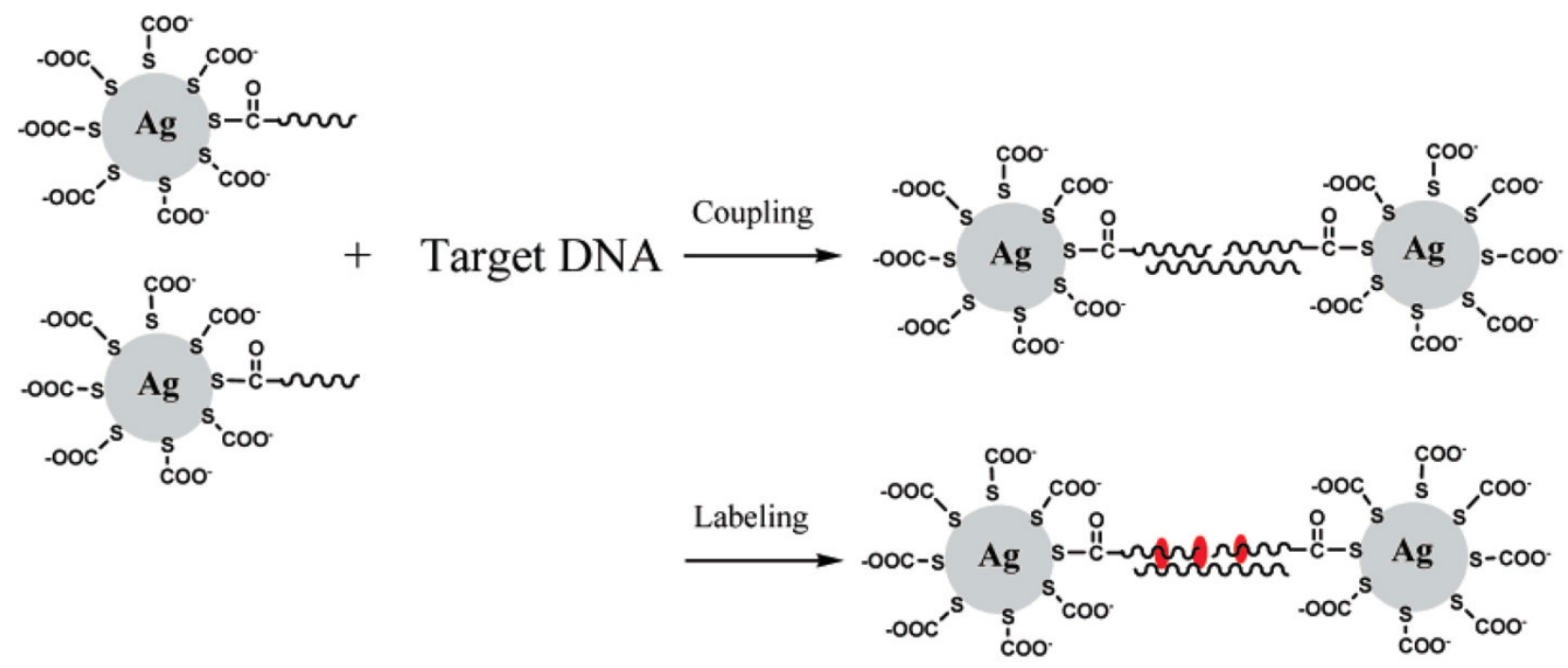

Aminated oligo: $\mathrm{H}_{2} \mathrm{~N}-3$ '-TCCACACACCACTGGCCATCTTG-5'

Target oligo: 3'-AGGTGTGTGGTGACCGGTAGAACTTTCAAGATGGCCAGTGGTGTGTGGA-5'

Scheme 1.

Coupling the Silver Particles by Hybridization with Target Oligonucleotide and Intercalating the YOYO into the DNA Duplex. 


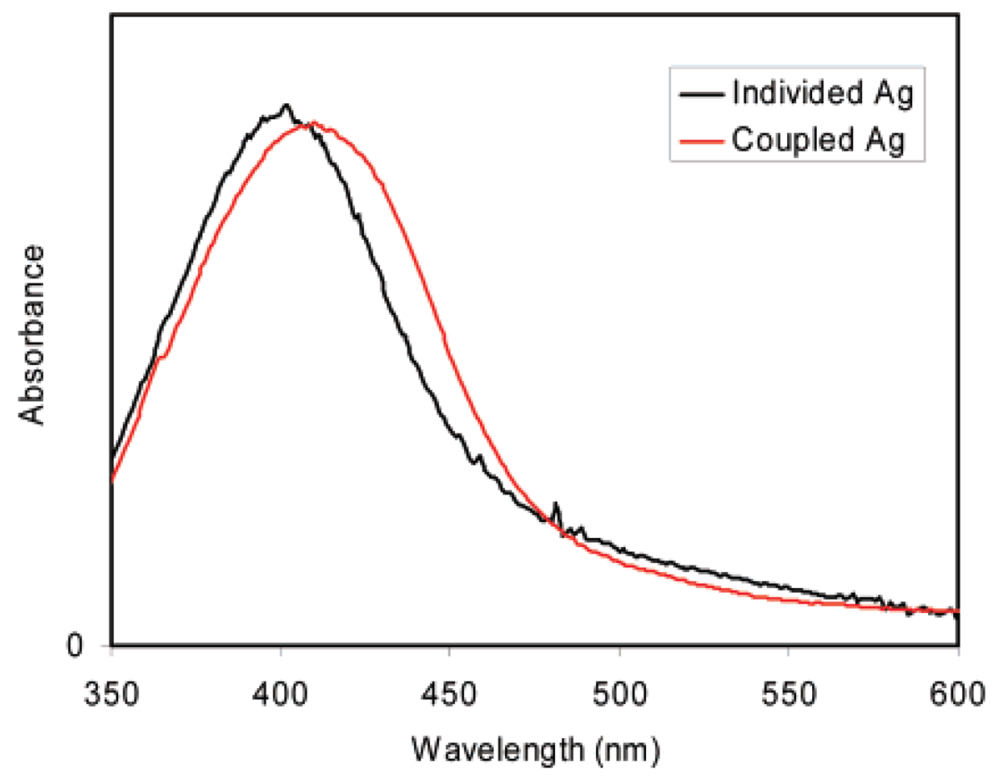

Figure 1.

Normalized absorbance spectra of individual and aggregated oligonucleotide-bound silver particles through hybridization with target complementary oligonucleotides. 
B

A
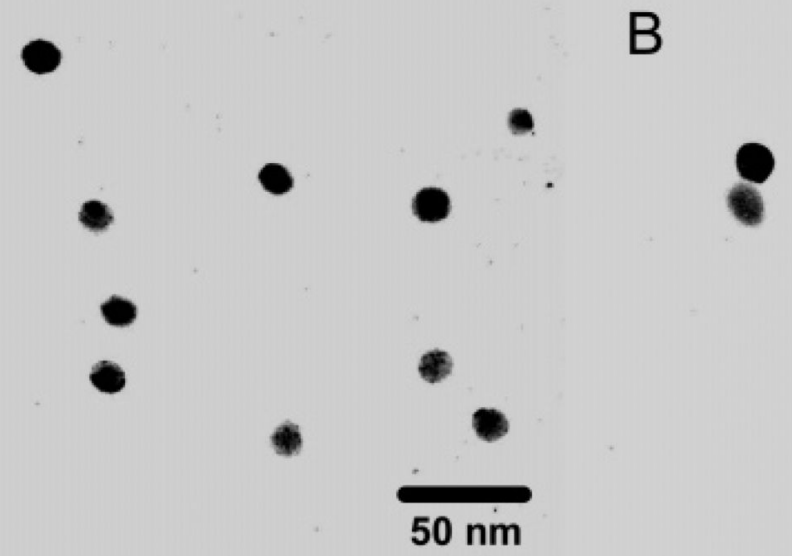

8
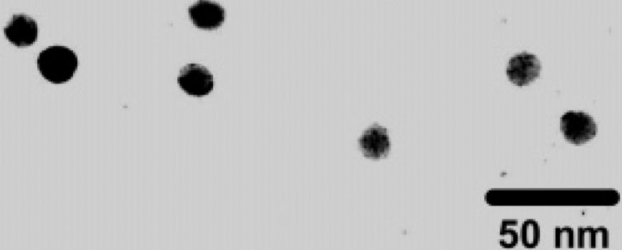

9

$50 \mathrm{~nm}$

Figure 2.

TEM images of (A) citrate-coated silver particles and (B) coupled silver particles by hybridization with target complementary oligonucleotides. 


\section{A: Free YOYO}

\section{B: DNA-YOYO}
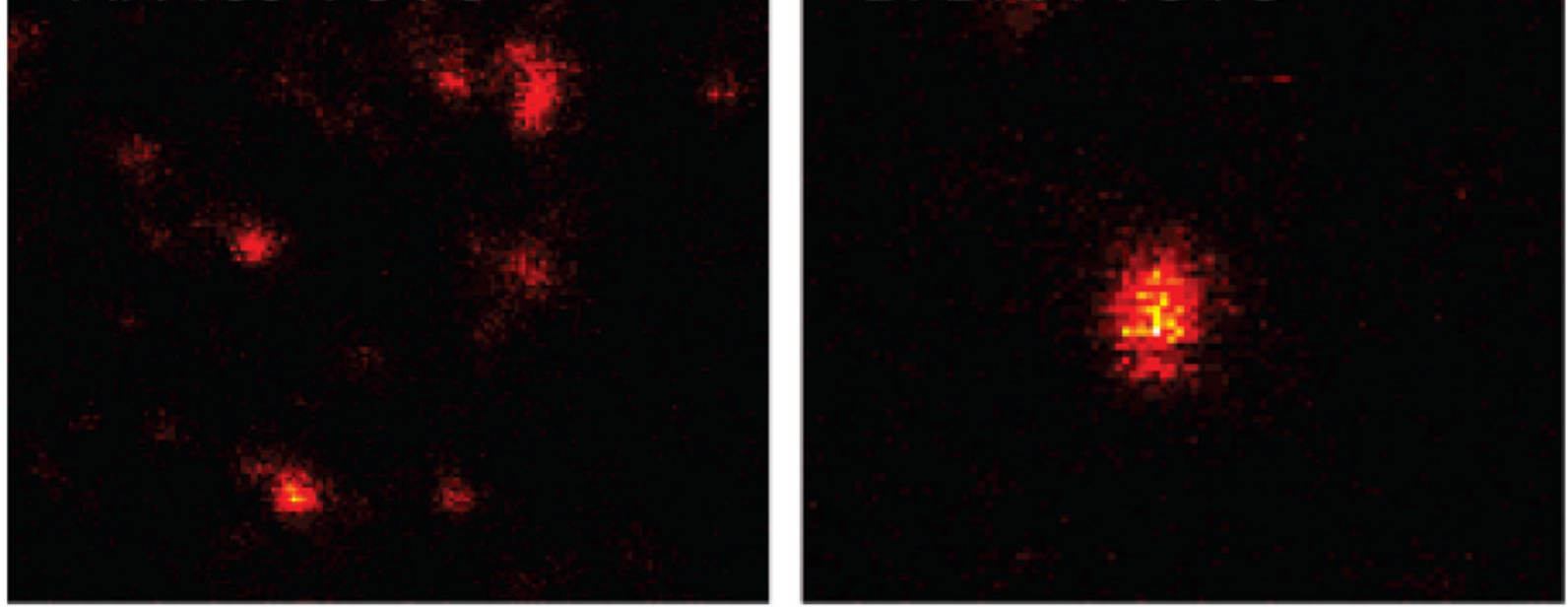

\section{C: Ag Scattering}
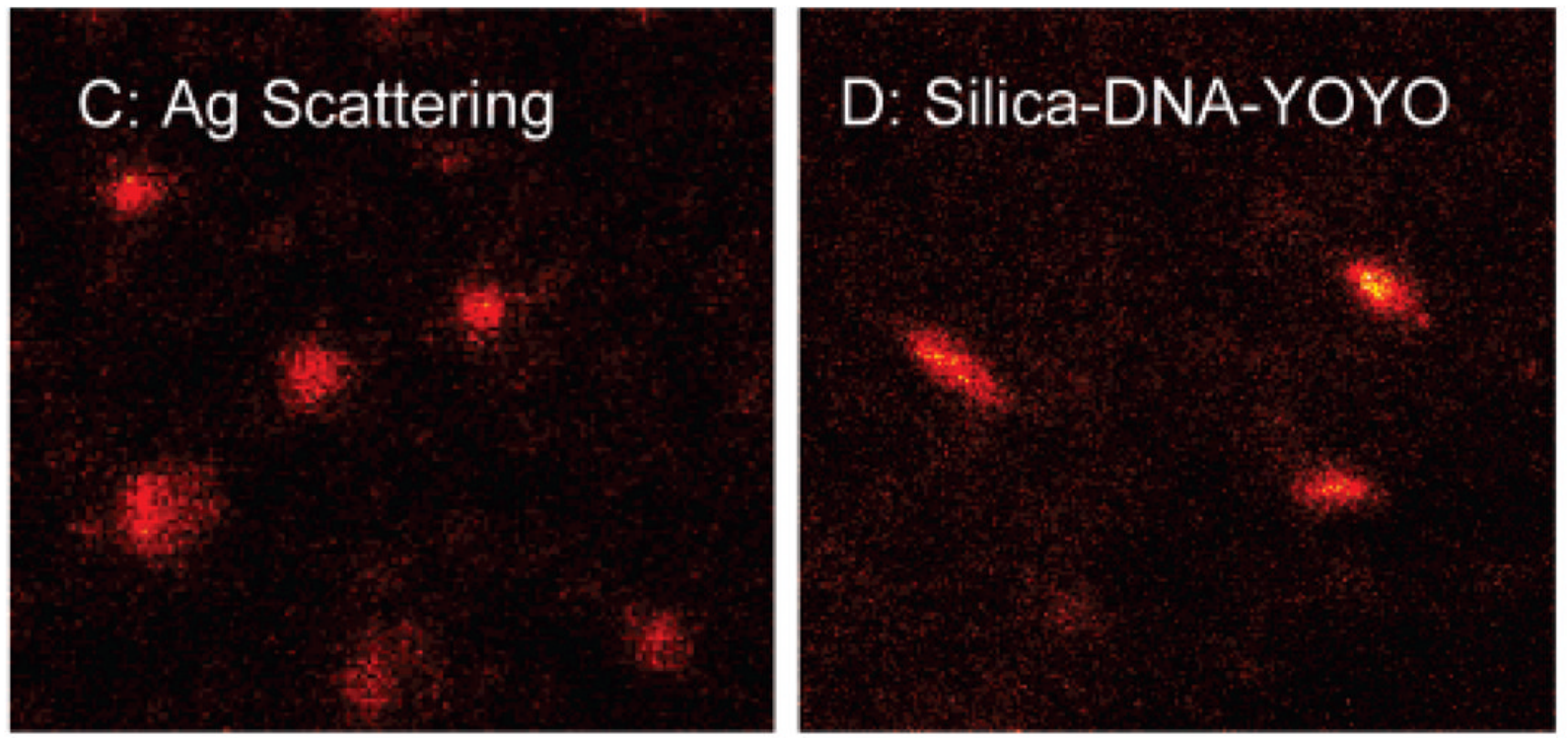

Figure 3.

Representative fluorescence images of (A) unbound YOYO fluorescence image, (B) silver particle scattering images, (C) YOYO intercalated into an unbound DNA duplex in the absence of metal, and (D) YOYO intercalated into a bound DNA duplex between silica beads. 


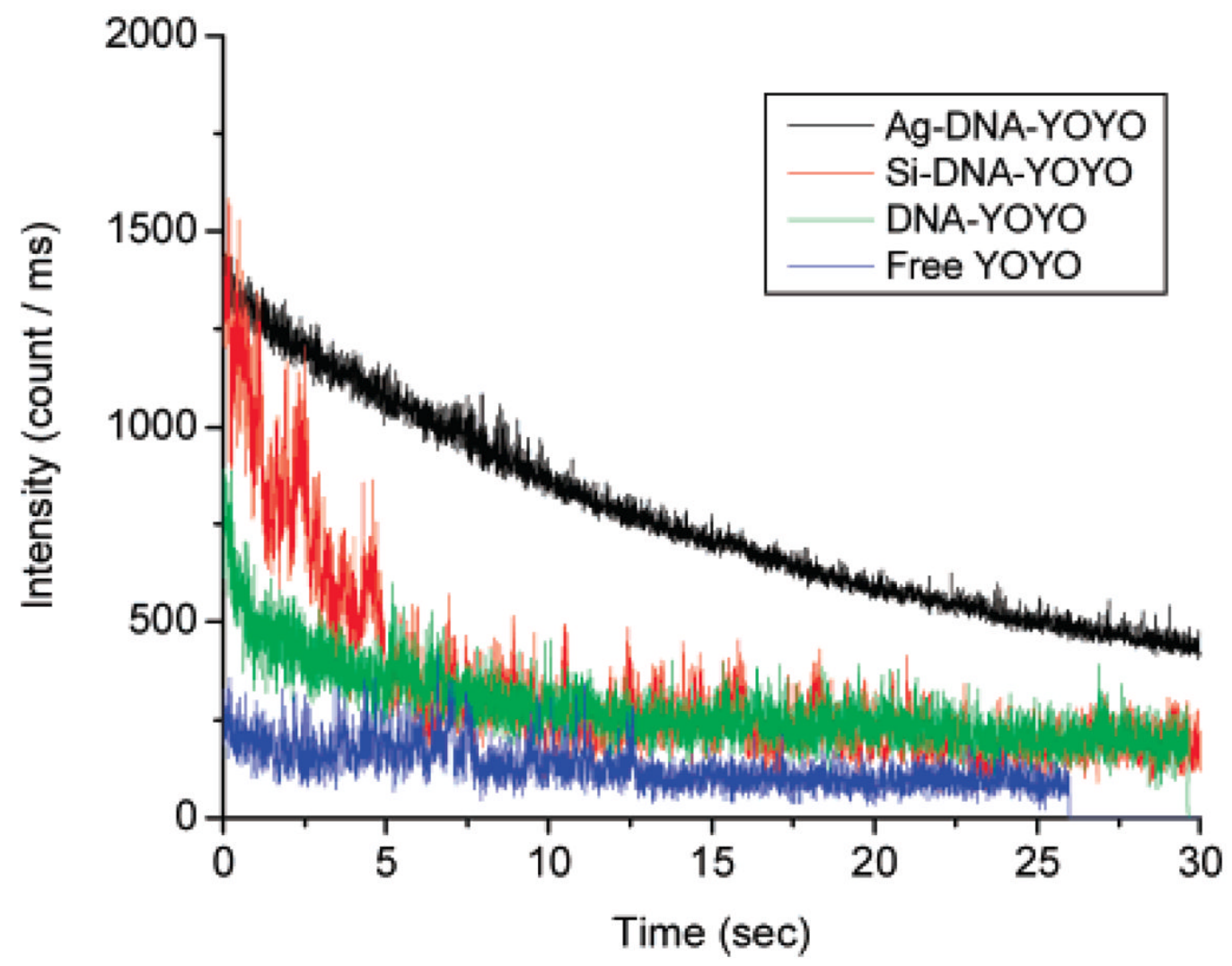

Figure 4.

Representative single-point fluorescence time transients for free YOYO, intercalate YOYO in an unbound DNA duplex, intercalated YOYO in a DNA duplex bound between coupled silica beads, and intercalated YOYO in a DNA duplex between coupled silver particles. 

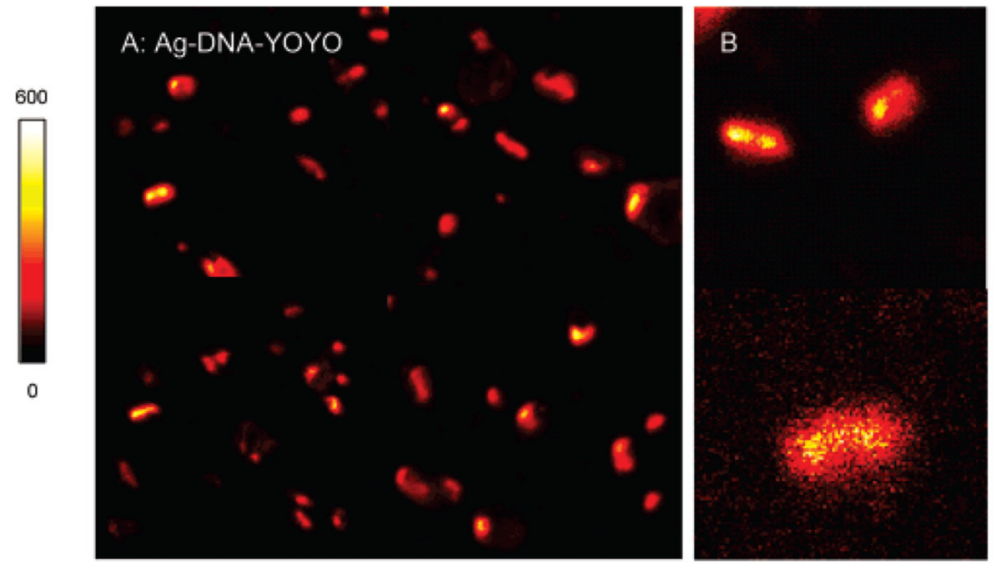

Figure 5.

Representative fluorescence images of (A) YOYO intercalated into a DNA duplex bound between silver particles and (B) the detail of these images. 


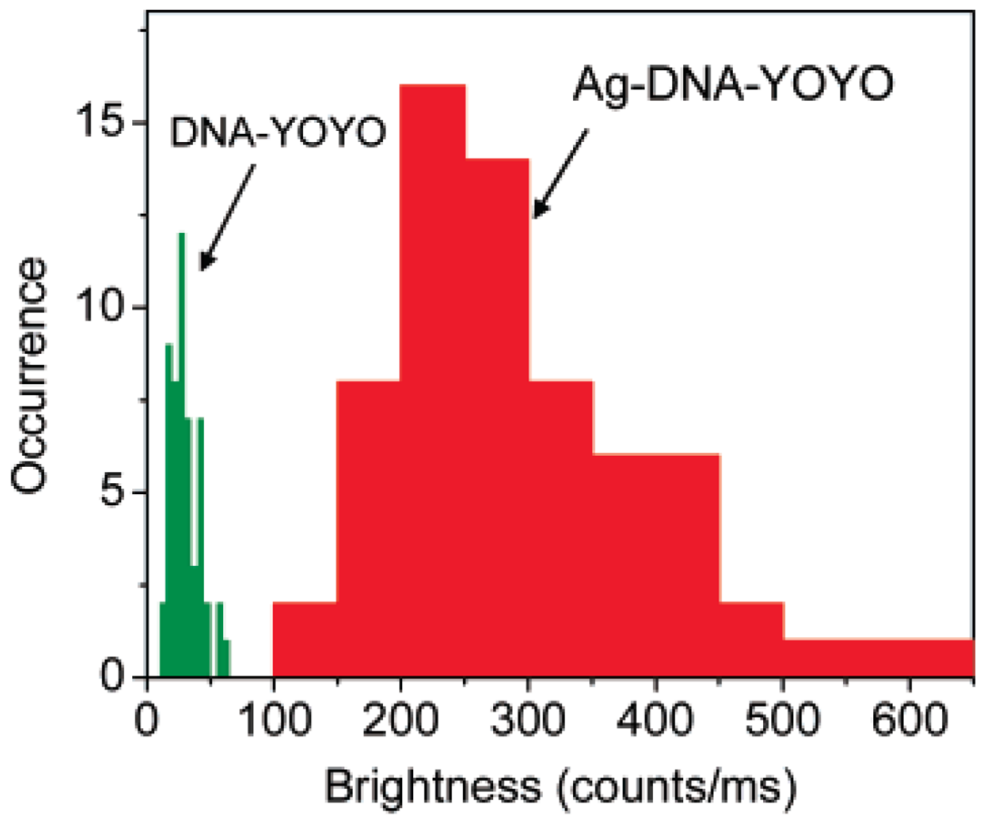

Figure 6.

Histograms of YOYO-labeled DNA in the absence of metal and between coupled silver particles. Note the intensity scale on the right is different from that in Figure 4. 


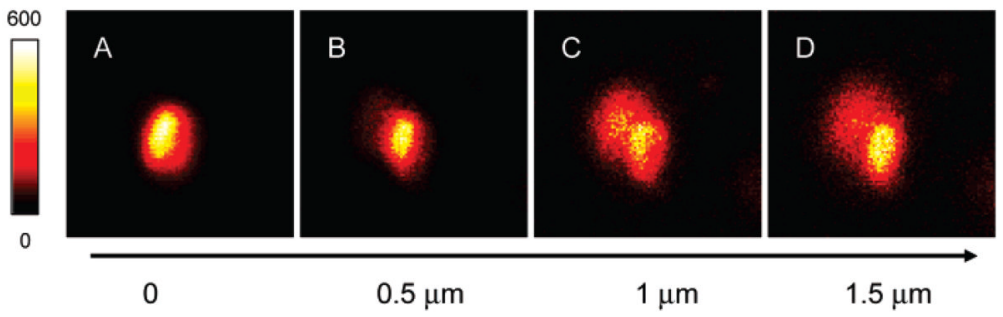

Figure 7.

Representative unfocused fluorescence images of YOYO intercalated into a DNA duplex bound between silver particles. 


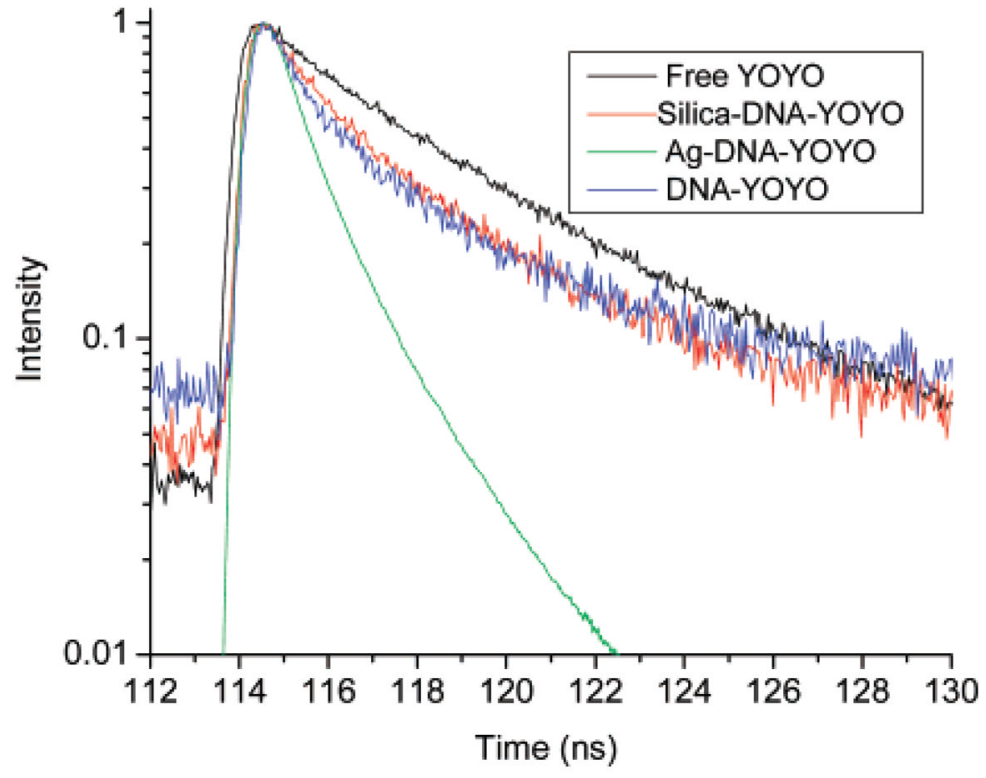

Figure 8.

Emission decay curves and fits for YOYO intercalated into DNA duplexes unbound or bound between silica beads or silver particles. 